\title{
"DIAGNÓSTICO DE LA CALIDAD DE LOS ACEITES DE OLIVA PRODUCIDOS EN LAS DIFERENTES ZONAS DE TACNA"
}

\author{
Responsable: Mgr. Liliana Lanchipa Bergamini \\ Miembro: Ing. Enrique De Florio Ramirez \\ Miembro: Mgr.Yolanda Sosa Gutierrez
}

\begin{abstract}
RESUMEN
Se estudiaron las características fisicoquimicas de los aceites de oliva de las variedades Leccino y Sevillana producidos principalmente en Tacna. Los resultados obtenidos nos indican que las muestras evaluadas corresponden a la categoría de aceite de oliva extra virgen. Los aceites obtenidos de la variedad Leccino muestran una acidez promedio de $0,13 \%$, indice de peróxidos de $1,97 \mathrm{meq} \mathrm{O} 2 / \mathrm{Kg}$. de aceite y un $\mathrm{K} 270$ de 0,1032 , y el de la variedad Sevillana y el de Leccino muestran una acidez promedio de 0,3\%, Índice de Peróxidos de 7,65 meq O2/ Kg., de aceite y un K270 de 0,1526
\end{abstract}

\section{ABSTRACT}

Physico-chemical characteristics of leccino, Sevillana, varieties of olive oil, produced principally in Tacna, were studied. The obtained results do not indicate that the evaluated samples are from the extra virgin olive oil. The obtained oil from the Leccino variety show that the average acidity index is $0,13 \%$ in oleic acid, the mean peroxide value is 1,97 meq $\mathrm{O} 2$ I Kg. Of oil and K270 from 0, 1032; and the Sevillana leccino oil show an average acidity of 0,3\% in oleic acid, the mean peroxide value is 7,65 meq $\mathrm{O} 2 / \mathrm{Kg}$. of oil, the mean $\mathrm{K} 270$ from $0,1526$.

\section{INTRODUCCIÓN}

El cultivo del olivo en nuestra ciudad y en el mundo se remonta a la antigüedad. El patrimonio olivicola y las producciones oleicolas relacionadas con este cultivo son apreciables, tanto desde el punto de vista económico - productivo como desde el punto de vista ambiental - geográfico. Las producciones que son también objeto de comercio internacional se caracterizan por un buen nivel cualitativo, no sólo en la producción de aceite de oliva sino también en el de la aceituna de mesa.

La producción de olivo en el Perú ha ido aumentando gradualmente, debido principalmente a que el Perú reúne muchas condiciones para este cultivo, en especial la zona sur, dada las ventajas climáticas que influyen en una mayor producción y rendimiento.

Las plantas que se dedican a la de extracción de aceite de oliva de la ciudad de Tacna han ido incrementando su producción, razón por la cual existe la necesidad de obtener productos de calidad que se encuentren enmarcados dentro de las exigencias que estipulan las normas nacionales e internacionales.

La mayoría de estos aceites se constituyen de una, dos hasta cinco variedades de aceituna, entre las que se pueden mencionar la Sevillana, Leccino, Empeltre y Frantoio como las más utilizadas.

La calidad del aceite de oliva virgen es el principal motivo de esta investigación; los estudios al respecto son pocos: es por ello que se vienen recopilando datos, año a año, sobre las características de calidad de estos aceites que se producen en nuestra ciudad para asi contribuir en la investigación preliminar de las características físicoquimicas de un producto propio de nuestra región.

\section{OBJETIVO}

Determinar la calidad del aceite de oliva virgen producido en Tacna.

\section{MATERIALESYMÉTODOS}

\subsection{Lugar de Ejecución}

El presente trabajo se ha realizado con la finalidad de evaluar la calidad de los aceites de oliva virgen que se producen en la ciudad de Tacna y determinar, por medio de las Normas Técnicas del INDECOPI así como normas de organismos internacionales, si se ajustan a los parámetros de calidad.

Los análisis se realizaron en los Laboratorios de Análisis Físico-Químico de la Facultad de Ingeniería en Industrias Alimentarias, y en el Laboratorio de Procesos y Operaciones Unitarias de la Universidad Nacional Jorge Basadre Grohmann de Tacna.

\subsection{Muestras, Reactivos, Materiales y Equipos:}

\subsubsection{Recolección de Muestras:}

Se seleccionaron muestras procedentes de distintas zonas productoras, se tomó en cuenta su aspecto general y su indice de madurez. 


\subsubsection{Reactivos:}

- Solución de Hidróxido de Sodio $(\mathrm{NaOH})$ $0,1 \mathrm{~N}$.

- Solución de Hidróxido de Potasio $(\mathrm{KOH}) 0,1 \mathrm{~N}$.

- Solución etanólica de Hidróxido de Potasio $(\mathrm{KOH}) 0,5 \mathrm{~N}$.

- SoluciónAlcohol Etilico-Éter Etílico.

- Solución alcohólica indicadora de Fenolftaleina (FF)al $1 \%$.

- Solución saturada de Yoduro de Potasio (IK).

- Solución indicadora de almidón.

- Solución de Ácido Acético-Cloroformo.

- Cloroformo.

\subsubsection{Materiales:}

- Matraces Erlenmeyer de 100 y $250 \mathrm{ml}$ con tapa esmerilada.

- Vasos de precipitado de $50 \mathrm{ml}$.

- Pipetas Graduadas de 1,5,10 ml.

- Pipetas Volumétricas de 0,5, 1 y $25 \mathrm{ml}$.

- Micropipetas desechables.

- Bureta automática graduada a $25 \mathrm{ml}$.

- Probetas de 25 y $50 \mathrm{ml}$.

- Fiolas de 25, 250 y $500 \mathrm{ml}$.

- Cápsulas de porcelana de fondo plano.

- Desecador.

- Picnómetros de $50 \mathrm{ml}$.

- Refrigerante de Reflujo

\subsubsection{Equipos:}

- Balanza analítica $\pm 0,0001 \mathrm{~g}$

- Espectrofotómetro UV-Visible $\pm 0,0001$

- Estufa de desecación

- Calefactor con agitador magnético

- RefractómetroAbbe

\subsection{Diseño del Experimento}

Para la realización del presente trabajo se han considerado aquellos productores y procesadores que tienen continuidad en la producción.

Las muestras se han analizado de acuerdo con los métodos analíticos propuestos por INDECOPI en la NTP 209.013 y métodos oficiales de análisis de aceites y grasas comestibles propuestos por $\mathrm{COI}$.

FIGURA 01: Diseño del Experimento

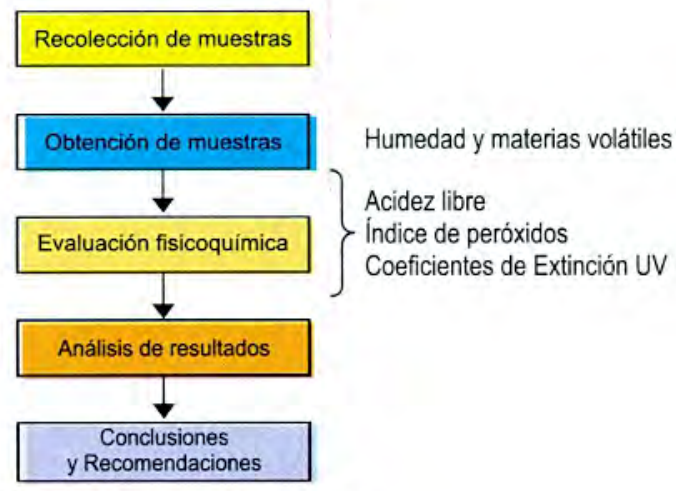

Fuente: Elaboración propia

\subsection{Técnicas y Métodos de Análisis}

2.4.1. Análisis Físicos - Químicos:

a) Determinación de Humedad y Materias Volátiles

Según Norma Técnica Nacional INDECOPI N 209.004 "Método para la determinación de la Humedad y materias volátiles.

\section{b) Determinación de la Acidez Libre}

Según la Norma Técnica Nacional INDECOPI 209.005. "Método para la Determinación de la Acidez Libre".

c) Determinación del Índice de Peróxido

Según la Norma Técnica Nacional INDECOPI 209.006. "Método para la determinación de Índice de Peróxido".

\section{d) Determinación de la Absorción UV}

Según el método COI/T20/Doc.19. Análisis Espectrofotométrico en el Ultravioleta. ParaAceites de oliva.

\section{RESULTADOS Y DISCUSIÓN}

\subsection{Humedad y Rendimiento Graso de las Aceitunas}

En esta etapa se recopilo los datos de la producción de aceite de oliva que se produce en Tacna para asi determinar las principales variedades que intervienen y que tienen mayor incidencia en el producto obtenido, determinándose que las variedades denominadas Sevillana entre las de mesa y Leccino (coratina) entre las aceiteras son las de mayor incidencia por variedad identificada en la composición del aceite obtenido.

CUADRO 01: Humedad y Rendimiento Graso de la Variedad Lección

\begin{tabular}{|l|c|c|c|c|}
\hline FECHA & $\begin{array}{c}\text { HUMEDAD } \\
\%\end{array}$ & $\begin{array}{c}\text { GRASA BASE } \\
\text { HUMEDA } \\
(\%)\end{array}$ & $\begin{array}{c}\text { GRASA BASE } \\
\text { SECA } \\
(\%)\end{array}$ & $\begin{array}{c}\text { RENDIMIENTO } \\
\text { INDUSTRIAL }\end{array}$ \\
\hline ABRIL & 56,56 & 16,32 & 37,57 & 15,87 \\
\hline MAYO & 55,81 & 19,24 & 43,54 & 17,43 \\
\hline JUNIO & 54,56 & 19,47 & 42,85 & 17,65 \\
\hline JULIO & 53,39 & 19,86 & 42,61 & 17,89 \\
\hline MEDIA & 55,08 & 18,725 & 41,6425 & 17,21 \\
\hline DESVEST & 1,3964 & 1,6219 & 2,7435 & 0,9128 \\
\hline COFVAR. & 2,5352 & 8,6632 & 6,588 & 5,3043 \\
\hline ERRORT & 0,6982 & 0,8109 & 1,3717 & 0,4564 \\
\hline
\end{tabular}

Fuente: Elaboración propia 
FIGURA 02:

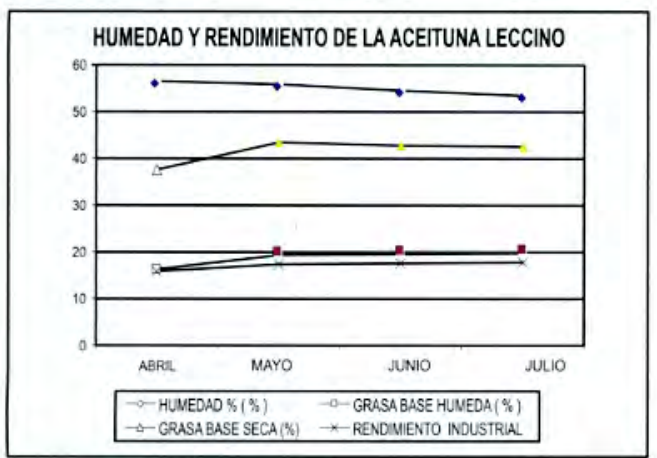

En el Cuadro 01 podemos observar el rendimiento graso de la variedad leccino presentando un promedio de $18,72 \%$ base húmeda, $41,64 \%$ en base seca y un rendimiento industrial promedio $17,21 \%$.

La humedad baja a lo largo de toda la campaña en la mayoría de los puntos de muestreo, desde $56,56 \%$ hasta $53,39 \%$.

El contenido de aceite sobre húmedo (GBH) aumenta durante la campaña desde $16,32 \%$ hasta $19,86 \%$,pues al descender la humedad aumenta el rendimiento sobre húmedo, pero el contenido real de aceite se observa en base seca donde no muestra una variación muy pronunciada sino, por el contrario, se mantiene casi constante.

En lo que se refiere al rendimiento industrial se puede observar que existe aproximadamente $1,51 \%$ de perdida a nivel industrial con esta variedad.

Esto se puede evidenciar mejor en las representaciones gráficas de los cuadros anteriormente mencionados (Figuras 02 y 03).

En el Cuadro 02 puede observarse que para la variedad Sevillana se observan valores más altos para la humedad obtenida, es asi como en marzo la aceituna presenta $60,13 \%$ en promedio de humedad para luego ir disminuyendo a lo largo del periodo de campaña hasta 57,13 que se han podido evaluar durante la primera semana de septiembre.

CUADRO 02: Humedad y Rendimiento Graso de la Variedad Sevillana

\begin{tabular}{|l|l|l|r|r|}
\hline FECHA & $\begin{array}{c}\text { HUMEDAD } \\
\% \\
(\%)\end{array}$ & $\begin{array}{l}\text { GRASABASE } \\
\text { HUMEDA } \\
(\%)\end{array}$ & $\begin{array}{l}\text { GRASA } \\
\text { BASE SECA } \\
(\%)\end{array}$ & $\begin{array}{l}\text { RENDIMIENTO } \\
\text { INDUSTRIAL }\end{array}$ \\
\hline MARZO & 60,13 & 12,32 & 30,9 & 8,67 \\
\hline ABRIL & 58,14 & 13,04 & 31,15 & 9,54 \\
\hline MAYO & 57,95 & 13,22 & 31,44 & 10,31 \\
\hline JUNIO & 57,97 & 17,86 & 42,49 & 12,48 \\
\hline JULIO & 57,71 & 18,43 & 43,58 & 13,62 \\
\hline AGOSTO & 57,99 & 18,86 & 44,89 & 13,37 \\
\hline SETIEMBRE & 57,96 & 19,73 & 46,93 & 1498 \\
\hline MEDIA & 5826428571 & 162086 & 38,7686 & 118529 \\
\hline DESV.EST & 0,8323 & 3,1933 & 7,2428 & 2,3614 \\
\hline COF.VAR. & 1,4286 & 19,7016 & 18,682 & 19,9226 \\
\hline ERRORT & 0,3146 & 1,2069 & 2,7375 & 0,8925 \\
\hline
\end{tabular}

Fuente: Elaboración propia

\section{FIGURA 03}

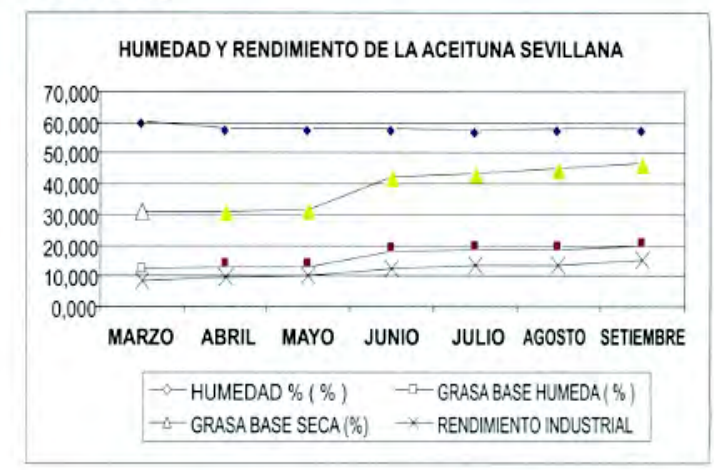

El rendimiento graso a nivel de laboratorio se inicia con un $12,32 \%$ de grasa en base húmeda y luego aumenta hasta $19,73 \%$. Comparando con el rendimiento a nivel industrial se puede observar un $4,3 \%$ en promedio de perdida en lo que se refiere al rendimiento, lo que se puede atribuir a varios factores pero entre los principales puede estar relacionado con la variedad y sistema de extracción.

\subsection{Características Químicas del Aceite de Oliva}

En este caso se debe mencionar que las muestras fueron obtenidas directamente de los tanques de almacenamiento.

CUADRO 03: Caraterizar Quimicas Iniciales del Aceite de Oliva Virgen

\begin{tabular}{|l|c|c|}
\hline PARAMETRO & SEVILLANA & LECCINO \\
\hline Acidez (\% de ácido oleico) & 0,3 & 0,13 \\
\hline Índice de Peróxidos (meq O2/kg & 7,65 & 1,97 \\
\hline K270 & 0,1526 & 0,1032 \\
\hline K232 & 1,378 & 1,3706 \\
\hline K225 & 1,69 & 2,0105 \\
\hline
\end{tabular}

Fuente: Elaboración propia

La acidez determina el contenido en ácidos grasos libres, presentes en un aceite, expresados en \% de ácido oleico por lo tanto la acidez mostrada inicialmente en un aceite recién obtenido es el resultado principal del mal estado de los frutos.

En este caso observamos en el Cuadro 03 que la acidez que presenta el aceite de la variedad Leccino es de 0,13 expresado en porcentaje de ácido oleico, y la acidez del aceite proveniente de la variedad Sevillana es de $0,30 \%$, lo cual nos indica que este aceite presenta un deterioro más pronunciado, evidenciando un mal manejo del fruto ya sea en la cosecha, postcosecha e inclusive el procesamiento.

Con respecto a los valores reportados en la Norma Técnica Nacional e Internacional (COI). estos valores corresponde a aceites extra virgenes.

El peróxido nos evidencia el grado de oxidación inicial del aceite. Los resultados obtenidos de este índice nos muestran con mayor 
magnitud la diferencia entre la oxidación del aceite de la variedad Leccino en la cual se reporta un valor de 1,97 meq $02 / \mathrm{kg}$ y el valor del aceite de la variedad Sevillana donde el valor es de 7,65 meq $\mathrm{O} 2$ $/ \mathrm{kg}$, casi cuatro veces mayor que el anterior.

Los indices observados son inferiores a 20 limite para el aceite de oliva extra virgen, lo que nos indica también que ambos aceites no presentan una importante oxidación inicial, asi como que tienen una buena aptitud y estado de conservación. En cuanto a los coeficientes de extinción al ultravioleta se determinaron con la finalidad de detectar la presencia de compuestos derivados de la oxidación

En el caso de la muestra de la variedad leccino el aceite presenta un valor para el K 270 de 0,1032 y en la de Sevillana 0,1526 lo cual comparándolas con los valores limites de acuerdo a la norma establecida por el Diario Oficial de Comunidades Europeas en el Reglamento (C.E.) 796/2002 que establece para el aceite oliva virgen extra el valor máximo aceptable en esta categoria es de 0,20 , se puede afirmar que estos aceites cumplen con la norma establecida.

Se presenta la misma situación en el caso de K232 siendo estas determinaciones muy importantes para determinar en este caso la medida de la oxidación primaria también si comparamos con la norma cumple lo exigido.

El K225 es un parámetro que nos indica el amargor y esta relacionado con el estado de madurez con respecto a las pruebas hechas los valores que se muestran están dentro del rango permitido.

\section{CONCLUSIONES}

1. De acuerdo con los resultados obtenidos con los indicadores señalados en la Norma Técnica Peruana ( NTP) 209.013 ITINTEC del INDECOPI y $\mathrm{COI}$, las muestras evaluadas corresponden a la categoria de aceite de oliva extra virgen.

2. La aceituna variedad Leccino presenta un rendimiento en Laboratorio de $18,72 \%$ y a nivel industrial un $17,21 \%$ que, comparado con la variedad Sevillana que presentó $\mathrm{m}$. Rendimiento a nivel de Laboratorio de $16,20 \%$ y a nivel industrial $11,85 \%$,podemos concluir que la aceituna de la variedad Leccino presenta mejor aptitud para el proceso tecnológico.

3. Los valores de la acidez en las muestras estudiadas son inferiores al $1 \%$, limite del grado de acidez para la categoría de aceite de oliva extra. Sin embargo, el aceite que provienen de la variedad Sevillana muestra un incremento de su acidez $(0,3 \%)$ mucho mayor que el de la leccino $(0,13 \%)$ atribuido a que esta variedad no es destinada en forma especifica a la extracción de aceite sino que resulta de saldos de las aceitunas que son dedicadas a mesa.

4. Los índices de peróxidos encontrados oscilan de 1,97 meq $02 / \mathrm{Kg}$ a $7,65 \mathrm{meq} 02 / \mathrm{Kg}$, inferiores al limite establecido por la norma de $20 \mathrm{meq} 02 / \mathrm{Kg}$ indicando que los aceites analizados no presentan una importante oxidación inicial. Para el caso del aceite Leccino muestra una mejor aptitud y estado de conservación frente a la Sevillana considerando que las condiciones de todo el manejo desde su obtención hasta su análisis respectivo fueron similares.

5. El K232 junto con el índice de peróxido se toman como medidas de la oxidación primaria $y$, en este caso, reafirmando la conclusión anterior evidenciamos que estos valores tanto para la Leccino como para el aceite procedente de la Sevillana, muestran valores por debajo del limite $(2,5)$ y muy similares. La oxidación secundaria evidenció a través del K270 que para las variedades Leccino y Sevillana fueron de 0,1032 y 0,1526 , respectivamente, y que estos valores, todavía no son muy significativos con respecto al limite permitido.

6. El K225 nos muestra un valor superior en lo que se refiere al aceite procedente de la variedad Leccino frente al de la Sevillana, evidenciando que éste presenta un amargor mucho más intenso, lo cual también nos favorece en su apreciación con respecto a la estabilidad que pudiera mostrar.

\section{BIBLIOGRAFÍA}

Anzaldua Morales, Antonio.1994, La evaluación sensorial de los alimentos en la teoría y practica. EditorialAcribia,S.A., Zaragoza, España, 1978.

Boskou, Dimitrios. 1998. Química y tecnología del aceite de oliva. $1^{\text {a }}$ ed. AMV Ediciones Mundi Prensa Libros. 191 p.

De La Rocha García, Germán. 1991. "Olivicultura Peruana. Cultivo y Procesamiento de Aceitunas". Ediciones CDPI-CIP. $1^{\text {a E} E d i c i o ́ n . ~ P e r u ́ . ~}$

, Germán. 1991. "Olivicultura Peruana. Cultivo y Procesamiento de Aceitunas". Ediciones CDPICIP. $1^{\text {a }}$ Edición. Perú.

Diario Oficial de Comunidades Europeas, 2002, L128/12-13.

Espinoza, E. 2002. Evaluación sensorial de los alimentos. Universidad Nacional Jorge Basadre Grohmann Tacna, Perù .188p.

Frias Ruiz L.; García-ortíz A.; Hermoso Fernández M.; Jiménez Márquez A.; Llavero Del

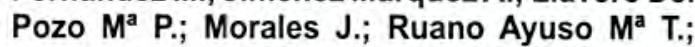
Uceda Ojeda M. 1999. Analistas de Laboratorio de Almazara. Colección Informaciones Técnicas 64/99. Junta de Andalucia. Comunidad Europea.

Instituto Nacional de Defensa de la Competencia y de la Protección de la Propiedad Intelectual (indecopi). 1991. Norma Técnica Peruana (NTP) 209.013 ITINTEC.

Kiritsakis, A.K. 1992. "El aceite de oliva". A. Madrid Vicente Ediciones. Madrid.

Madrid Vicente, Cenzano Del Castillo, Madrid Cenzano. 1997. "Manual de aceites y grasas comestibles". Capítulo VII.

Pedrero F. Daniel Y Pangborn Rose Marie, 1989. Evaluación sensorial de los alimentos, métodos analiticos. Editorial Alambra Mexicana,S.A, 251p.

Tous Martl, Joan; ROMERO AROCA, Agustí. 1992. Variedades del olivo. Con especial referencia a Cataluña. Fundación "la Caixa".

Ureña Peralta Miber O. y Diarrigo H. Matilde. 1999. Evaluación sensorial de Los Alimentos. $1^{\text {a }}$.ed. Editorial Agraria. Lima. 210p. 